\title{
ARTICLE OPEN \\ Biofilm-associated bacterial amyloids dampen inflammation in the gut: oral treatment with curli fibres reduces the severity of hapten-induced colitis in mice
}

\author{
Gertrude O Oppong ${ }^{1}$, Glenn J Rapsinski ${ }^{1}$, Sarah A Tursi ${ }^{1}$, Steven G Biesecker ${ }^{1}$, Andres JP Klein-Szanto ${ }^{2}$, Mark Goulian ${ }^{3}$, \\ Christine McCauley ${ }^{4}$, Catherine Healy ${ }^{4}$, R Paul Wilson $^{1}$ and Cagla Tükel ${ }^{1}$
}

\begin{abstract}
BACKGROUND/OBJECTIVES: A disruption of epithelial barrier function can lead to intestinal inflammation. Toll-like receptor (TLR) 2 activation by microbial products promotes intestinal epithelial integrity and overall gut health. Several bacterial species, including enteric bacteria, actively produce amyloid proteins as a part of their biofilms. Recognition of amyloid fibres found in enteric biofilms, termed curli, by the Toll-like receptor (TLR)2/1 complex reinforces barrier function. Here, we investigated the effect of purified curli fibres on inflammation in a mouse model of acute colitis.

METHODS: Bone marrow-derived macrophages as well as lamina propria cells were treated with curli fibres of both pathogenic Salmonella enterica serovar Typhimurium and commensal Escherichia coli Nissle 1917 biofilms. Mice were given 0.1 or 0.4 mg of purified curli orally 1 day post administration of 1\% 2,4,6-trinitrobenzene sulphonic acid (TNBS) enema. Histopathological analysis was performed on distal colonic tissue taken 6 days post TNBS enema. RNA extracted from colonic tissue was subjected to RT-PCR. RESULTS: Here we show that curli fibres of both pathogenic and commensal bacteria are recognised by TLR2 leading to the production of IL-10, immunomodulatory cytokine of intestinal homeostasis. Treatment of mice with a single dose of curli heightens transcript levels of I/10 in the colon and ameliorates the disease pathology in TNBS-induced colitis. Curli treatment is comparable to the treatment with anti-tumour necrosis factor alpha (anti-TNFa) antibodies, a treatment known to reduce the severity of acute colitis in humans and mice.

CONCLUSION: These results suggest that the bacterial amyloids had a role in helping to maintain immune homeostasis in the intestinal mucosa via the TLR2/IL-10 axis. Furthermore, bacterial amyloids may be a potential candidate therapeutic to treat intestinal inflammatory disorders owing to their remarkable immunomodulatory activity.
\end{abstract}

npj Biofilms and Microbiomes (2015) 1, 15019; doi:10.1038/npjbiofilms.2015.19; published online 14 October 2015

\section{INTRODUCTION}

The human gastrointestinal (GI) immune system encounters an estimated one hundred trillion bacteria representing more than 1,000 species. $^{1-3}$ A large percentage of these populations of bacteria live in the distal $\mathrm{Gl}$ tract. ${ }^{4}$ Once thought of as passive participants in $\mathrm{Gl}$ homeostasis, it is now known that the microbiota are actively involved in initiating immune responses that contribute to $\mathrm{Gl}$ immune cell development and homeostasis. Recognition of gut microbiota and microbiota-derived components via pattern recognition receptors (PRRs) help maintain mucosal barrier integrity. Toll-like receptor (TLR) 2 has been widely discussed in the literature to have a pivotal role not just in maintaining intestinal epithelial integrity but also in mediating immune responses that promote overall gut health. ${ }^{5-8}$ TLR2deficient mice are more susceptible to bacteria-induced ${ }^{9}$ and chemically induced colitis. ${ }^{6}$ Moreover, TLR2 recognition of commensal bacteria is required for intestinal homeostasis. ${ }^{8}$ TLR2 signalling depends on the adaptor molecules MyD88 or TIRAP/ MAL resulting in a pro- or anti-inflammatory outcome. ${ }^{5,10,11}$ In the intestinal mucosa, TLR2 mostly regulates the anti-inflammatory responses and the reinforcement of the epithelial barrier. Recruitment of the PI3K-Akt to the MyD88/MAL complex upon
TLR2 activation results in the expression of anti-inflammatory cytokines including IL-10 and repression of the pro-inflammatory pathway. ${ }^{12}$ Furthermore, activation of PI3K-Akt leads to the expression of tight junction proteins such as ZO-1 in epithelial cells. $^{6,7}$ IL-10 has been implicated to have a vital role in maintaining gut homeostasis. In fact, IL-10-deficient mice raised in conventional animal housing develop spontaneous enterocolitis by $4-8$ weeks of age. ${ }^{13}$

Lipoproteins from Gram-negative bacteria are canonical TLR2/1 ligands. ${ }^{14}$ However, lipoproteins are buried in the outer membrane and stimulation of the TLR2/1 receptor complex by intact $E$. coli cells is dominated by curli, ${ }^{15}$ an amyloid secreted to the extracellular matrix of biofilm by both commensal and pathogenic members of the Enterobacteriaceae. ${ }^{16-19}$ Curli fibres, initially described in S. Typhimurium as thin aggregative fimbriae, are encoded by the csg gene cluster that consist of csgBAC and csgDEFG genes. ${ }^{20}$ The major subunit of curli, $\operatorname{CsgA}$, is transcribed from the $\operatorname{csg} A$ gene under the control of the master regulator $\mathrm{CsgD}^{21}$ Although $\mathrm{CsgA}$ has the intrinsic propensity to self-aggregate, it requires the $\mathrm{CsgB}$ subunit for nucleation into fibres. ${ }^{22-24}$ Curli-like proteins have been found within four phyla; Bacteroidetes, Proteobacteria, Firmicutes and

\footnotetext{
${ }^{1}$ Department of Microbiology and Immunology, School of Medicine, Temple University, Philadelphia, PA, USA; ${ }^{2}$ Department of Pathology, Fox Chase Cancer Center, Philadelphia, PA, USA; ${ }^{3}$ Department of Biology, University of Pennsylvania, Philadelphia, PA, USA and ${ }^{4}$ Janssen Research \& Development, LLC, Spring House, PA, USA. Correspondence: C Tükel (ctukel@temple.edu)
}

Received 17 April 2015; revised 2 September 2015; accepted 7 September 2015 
Thermodesulfobacteria. ${ }^{18,25}$ Curli are an essential proteinaceous component of the extracellular biofilm matrix that allows enteric bacteria to bind to biotic and abiotic surfaces. ${ }^{26-28}$ We recently determined that the innate immune recognition of curli is mediated by TLR2/1 heterocomplex. ${ }^{15,29,30}$ Recognition of curli fibres by TLR2 complex leads to the augmentation of the intestinal epithelial barrier and limits bacterial translocation from the intestinal lumen during infection. ${ }^{31}$ In addition, the NLRP3 inflammasome cooperates with the TLR2/1 receptor complex to recognise curli fibres leading to the production of $\mathrm{IL}-1 \beta .^{32}$ Interestingly, both TLR2 and NLRP3 have been implicated for the recognition of human amyloids. In addition to the latter two receptors, recently formyl peptide receptor 2 was identified as a receptor for human amyloids, amyloid beta and serum amyloid A, and the bacterial amyloid, phenol soluble modulins (PSM) of Staphylococcus aureus. ${ }^{33-36}$

Curli fibres possess a cross $\beta$-sheet quaternary structure that is characteristic of amyloids in general and makes these proteins resistant to proteolytic digestion, ${ }^{18,20,37}$ a property that should facilitate intact transition through the $\mathrm{Gl}$ tract. We thus investigated whether oral administration of this TLR2 ligand would ameliorate inflammation in a mouse model of colitis.

\section{MATERIALS AND METHODS}

Bacterial strains and culture conditions

E. coli Nissle 1917 (ECN) was first described by physician Alfred Nissle. ${ }^{38}$ Salmonella enterica serovar Typhimurium (denoted as STM) msbB mutant (RPW3) was described previously. ${ }^{39}$ Bacterial growth was supported with $50 \mu \mathrm{g} / \mathrm{ml}$ of kanamycin or $100 \mu \mathrm{g} / \mathrm{ml}$ nalidixic acid where needed.

\section{Purification of curli fibres}

To induce the production of curli fibres, $500 \mu$ l of the overnight culture of bacteria was inoculated into $200 \mathrm{ml}$ of YESCA broth supplemented with $4 \%$ dimethylsulphoxide (ACROS) at $28^{\circ} \mathrm{C}$ for $72 \mathrm{~h}$ with shaking at 200 r.p.m. ${ }^{40}$ Purification of curli fibres from the $S$. Typhimurium $m s b B$ mutant (RPW3) or $\mathrm{EcN}$ was performed according to an established protocol. ${ }^{41}$ Briefly, bacterial cells were collected and subjected to multiple treatments of lyzozyme, DNAse and RNAse as well as boiling in SDS. Samples were run overnight in a $12 \%$ SDS-preparative gel and insoluble curli fibres were recovered from the well.

\section{Cell/tissue culture}

Bone marrow-derived macrophages. Bone marrow-derived macrophages were generated from age-matched C57BL/6 or TLR2 - / - (approximately 6-8 weeks) female mice as described previously. ${ }^{29}$ Supernatants were assayed for IL-10 by ELISA (eBioscience, Hatfield, UK). Experiments were repeated three times.

Lamina propria cells. Lamina propria (LP) cells were isolated using the Lamina Propria Dissociation Kit (mouse) from Miltenyi Biotec (Bergisch Gladbach, Germany). The LP cells were processed from whole-mouse small and large intestine using the company's suggested protocol. The experiments were repeated three times.

Transfection of HeLa cells. Transfection studies using HeLa cells and the vectors were described previously. ${ }^{15}$ The cells were stimulated with $2.5 \mathrm{\mu g} / \mathrm{ml}$ purified curli, GST, GST-CsgA or $\mathrm{Pam}_{3} \mathrm{CSK}_{4}$ (Invivogen, San Diego, (A, USA).

\section{Experimental colitis}

The 2,4,6-trinitrobenzenesulfonic acid (TNBS)-induced colitis model is a wellcharacterized animal model of inflammatory bowel disease. ${ }^{42}$ Briefly, groups of six to seven 4-6-week Balb/cJ mice were starved $24 \mathrm{~h}$ before receiving an enema containing $60 \mu \mathrm{l}$ of $50 \%$ ethanol solution (mock) or a $1 \%$ TNBS solution in $50 \%$ ethanol intrarectally approximately $3-\mathrm{cm}$ into the colon. A day after TNBS colitis induction, mice were given the following treatments (per mouse): 0.1 or $0.4 \mathrm{mg}$ curli fibres in $100 \mu \mathrm{l}$ of water or $100 \mu \mathrm{l}$ sterile water as a mock via oral gavage or $100 \mu \mathrm{g}$ of anti-tumour necrosis factor alpha (anti-TNFa) via intraperitoneal injection as a positive control. Animals were monitored daily for weight/physical changes for 3 days or 6 days. On day 3 or 6 post TNBS induction, mice were euthanized via carbon dioxide asphyxiation. Samples from the colon were collected for messenger RNA and histopathology analysis. Colitis experiments were repeated three times. Sample size for this experiment was chosen to obtain statistical significance between groups on the basis of statistical power calculations $\left(n=2 \mathrm{SD}^{2}\left(Z^{-3}+Z\right)^{2} / \mathrm{d}^{2}\right)$, previous experience and literature review. No randomisation was used. Colitis experiments were approved by Temple University's Institutional Animal Care and Use Committee.

\section{Histopathology}

Tissue samples from colon were harvested immediately following euthanasia and were fixed with $10 \%$ buffered formalin. Colon tissue samples were cut and stained with hematoxylin and eosin at the Fox Chase Cancer Center Histopathology Core Facility. Colitis was scored independently and blindly by a pathologist.

\section{Real-time PCR}

To examine messenger RNA levels of II- 10, Tgf- $\beta,{ }^{43}$ Ifn- $\gamma_{,}{ }^{44} \mathrm{Tnfa}^{45}$ and II- $6,{ }^{44}$ tissue samples from mice were taken at the time of euthanasia and immediately frozen in liquid nitrogen for further processing. RNA was isolated from the tissue using the Qiagen RNeasy kit according to company specifications. Following RNA isolation, $1 \mu \mathrm{g}$ of RNA was reverse transcribed using murine leukaemia virus reverse transcriptase (Applied Biosciences). Complementary DNA (2.5 $\mu$ l) was subjected to quantitative PCR amplification using SYBR Green (Applied Biosystems, Waltham, $M A, U S A)$ and the primers listed above. Fold change differences in amplification were determined using the $\Delta C_{\mathrm{T}}$ approach with $G a p d h^{46}$ as the housekeeping gene.

\section{Statistical analysis}

Differences between data sets were analysed using the Student's $t$-test ( $P$ value $<0.05$ is significant) where applicable. For the analysis of bacterial numbers, values were logarithmically converted before statistical analysis. Histopathological scores were analysed using the Mann-Whitney test.

\section{RESULTS}

II-10 is induced in bone marrow-derived macrophages in response to curli fibres in a TLR2-dependent manner

Curli fibres are produced by commensal and pathogenic strains of Enterobacteriaceae family members including $E$. coli and $S$. Typhimurium. Our previous studies have established that curli fibres from $S$. Typhimurium are recognised by TLR2 (refs $15,29,30,47,48$ ) and specifically the TLR2/1 heterocomplex recognises both the purified curli as well as the recombinant GST-tagged CsgA (Figures 1a and b). Previous studies had shown that a commensal strain of E. coli Nissle 1917 (EcN), dampens inflammation through the production of IL-10 via TLR2 activation in the GI tract. ${ }^{49,50}$ Triacylated lipoproteins from Gram-negative bacteria are canonical TLR2/1 ligands (Supplementary Figure S1). However, as EcN also expresses curli fibres (Supplementary Figure S2), the mechanism by which the EcN triggers TLR2 activation in the gut remains unknown. Thus, we first wanted to compare the stimulatory activities of curli fibres from pathogenic vs commensal bacteria and determine whether curli fibres induced IL-10 production in immune cells. We used TLR2 - /mice that are on C57BL/6 mice background. Stimulation with curli fibres purified from EcN elicited induction of IL-10 in bone marrow-derived macrophages from C57BL/6 mice, whereas this response was blunted in macrophages derived from TLR2 - / mice (Figure 1c). Similar results were obtained when the experiment was repeated with curli purified from an $S$. Typhimurium $m s b B$ mutant, which expresses a modified lipid A that does not signal through TLR4, ${ }^{51}$ suggesting that LPS did not markedly contribute to the observed responses. Furthermore, macrophages produced similar levels of IL- 6 in response to curli fibres from both species in a TLR2-dependent manner (Supplementary Figure S3). 

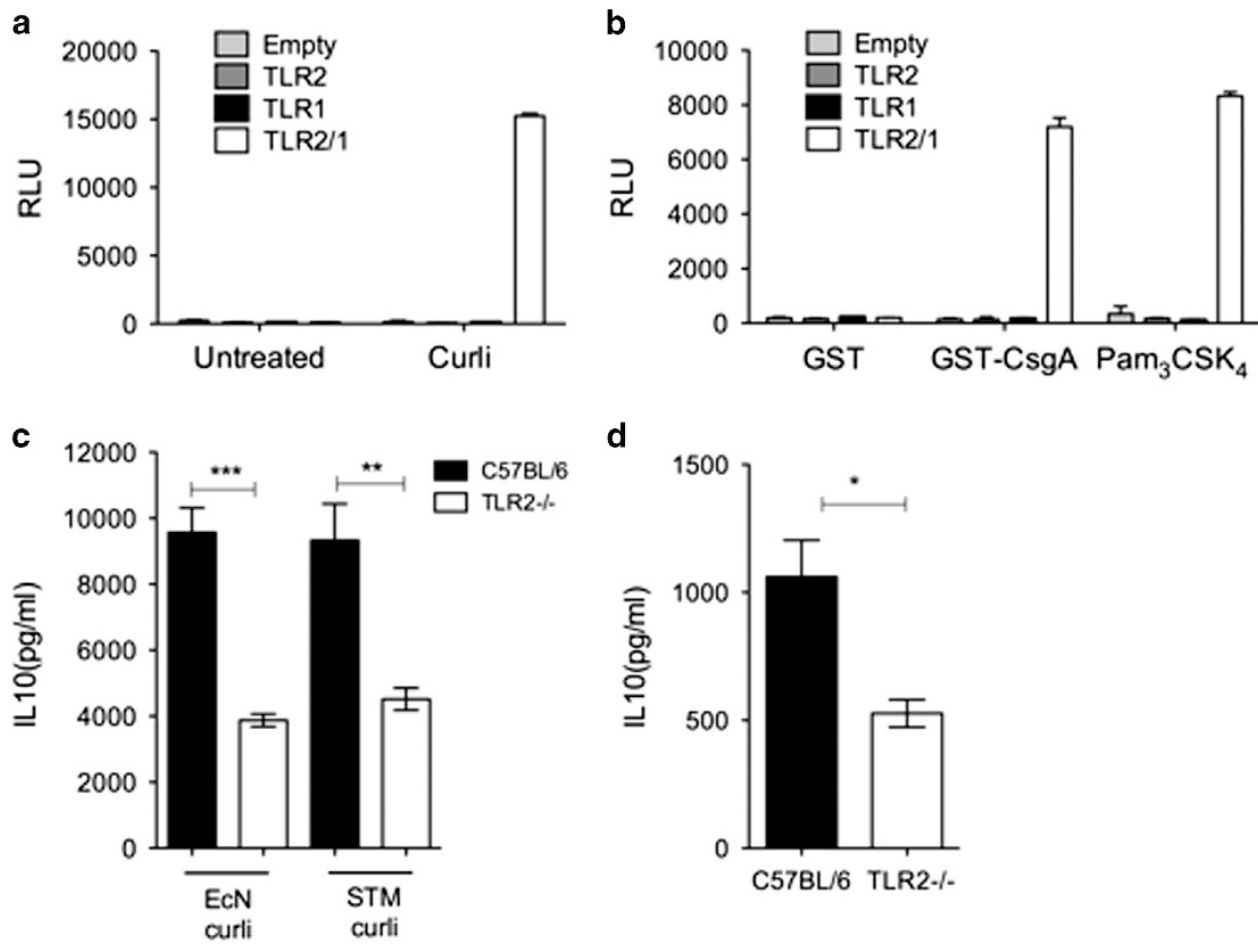

Figure 1. Curli fibres from a commensal and pathogen induce cytokines from bone marrow-derived macrophages in a TLR2-dependent manner. HeLa cells carrying a NFkB luciferase reporter were mock-transfected (vector) or transfected with TLR1, TLR2 or TLR2/TLR1. Cells were stimulated with (a) $2.5 \mu \mathrm{g} / \mathrm{ml}$ purified curli or (b) GST-CsgA fusion protein or synthetic tri-acylated lipopeptide $\left(\mathrm{Pam}_{3} \mathrm{CSK}_{4}\right)$. Untreated cells or cells stimulated with GST protein served as negative controls. Activity of the NFKB luciferase reporter activity was measured and expressed as relative luminescence units (RLU). (c) Bone marrow-derived macrophages (BMDMs) from 6 to 8 -week-old female C57BL/6 ( $n=3)$ and TLR2 $-/-(n=3)$ old mice were treated with $5 \mu \mathrm{g} / \mathrm{ml}$ of curli fibres isolated from biofilms of STM or EcN for $24 \mathrm{~h}$. Supernatants were collected and IL-10 protein levels were measured using ELISA. IL-10 was not detected in samples that were unstimulated (control). (d) Lamina propria (LP) cells were isolated from the intestine of C57BL/6 $(n=3)$ and TLR2 - / $(n=3)$ mice as described in the Materials and Methods. LP cells were incubated with $5 \mu \mathrm{g} / \mathrm{ml}$ curli fibres for $24 \mathrm{~h}$. Supernatants were then collected and assayed for IL- 10 by ELISA. $\left({ }^{*} P\right.$ value $<0.05 ; 95 \%$ confidence interval). STM, Salmonella enterica serovar Typhimurium. ELISA, enzyme-linked immunosorbent assay.

As TLR2 has been reported previously to be an essential receptor for Gl homeostasis and repair, ${ }^{5-7,9}$ we investigated the interaction of curli fibres with cells of the Gl tract by treating the intestinal LP cells isolated from the colonic tissue of C57BL/6 mice and TLR2 - / - mice with purified curli fibres. Consistent with what we had noted with bone marrow-derived macrophages, ex vivo treatment of intestinal cells with curli fibres resulted in the production of IL-10 in a TLR2-dependent manner (Figure 1d). Overall, these results indicated that curli fibres are recognised by TLR2, which results in the production of IL-10.

Oral administration of curli fibres in non-colitic mice does not elicit cytokine production

The $\beta$-sheet structure of amyloids makes them resistant to chemical as well as enzymatic treatments. In fact, amyloids only dissociate into their monomers by treatment with $90 \%$ formic acid or hexafluoroisopropanol treatment in vitro. ${ }^{52}$ Thus, amyloids may be resilient to conditions encountered in the intestinal lumen. Consistent with this idea, curli fibres can be detected in faecal matter for up to $12 \mathrm{~h}$ after oral administration (Supplementary Figure S4). To test the effect of curli fibres on mucosal gene expression in the intestine, we treated $\mathrm{C} 57 \mathrm{BL} / 6$ mice with a cocktail of antibiotics including ampicillin $(1 \mathrm{~g} / \mathrm{l})$, neomycin $(1 \mathrm{~g} / \mathrm{l})$, metronidazole $(1 \mathrm{~g} / \mathrm{l})$ and vancomycin $(0.5 \mathrm{~g} / \mathrm{l})$ for 7 days in drinking water to mimic germ-free conditions. ${ }^{53}$ Following intensive antibiotic administration, $0.1 \mathrm{mg}$ curli fibres were administered via oral gavage and cytokine production was determined in the intestinal tissue from these mice using quantitative RT-PCR. There were no significant changes in I/10, Ifng, Tgfb or $1 / 6$ messenger RNA expression between the treatment and control groups $72 \mathrm{~h}$ post administration (Figure 2) suggesting that curli fibres do not elicit significant changes in cytokine expression during steady-state conditions in the gut.

Intraperitoneal injection of curli fibres induces interleukin-10 expression in mouse small intestinal tissue

Systemic administration of flagellin induces changes in gene expression in intestinal epithelial cells and Paneth cells along the entire length of the small intestine. ${ }^{54}$ As the administration of curli fibres via oral gavage of mice with an intact epithelial barrier did not elicit detectable immune responses (Figure 2), we wanted to investigate whether the immune cells underlying the LP have an important role in responses to curli. We thus injected C57BL/6 or TLR2 - / - mice intraperitoneally with the curli fibres. Seventy-two hours after injection, we determined the gene expression profiles in the small intestine and spleen by quantitative RT-PCR. Interestingly, /110 expression was upregulated in the small intestinal tissue from C57BL/6 mice at $72 \mathrm{~h}$. Remarkably, the expression of $/ 110$ was blunted significantly in TLR2 - / - mice. No upregulation of pro-inflammatory cytokines Ifng, II6 or another immunomodulatory cytokine Tgfb was observed in the small intestinal tissue (Figure 3a). Intriguingly, a completely different pattern of cytokine expression was observed in the spleen. Although we did not detect $1 / 10$, Tgfb or $1 / 6$, transcripts for Ifng were induced by curli in a TLR2-dependent manner (Figure 3b). These data suggested that (i) the subepithelial LP cells have an 
4
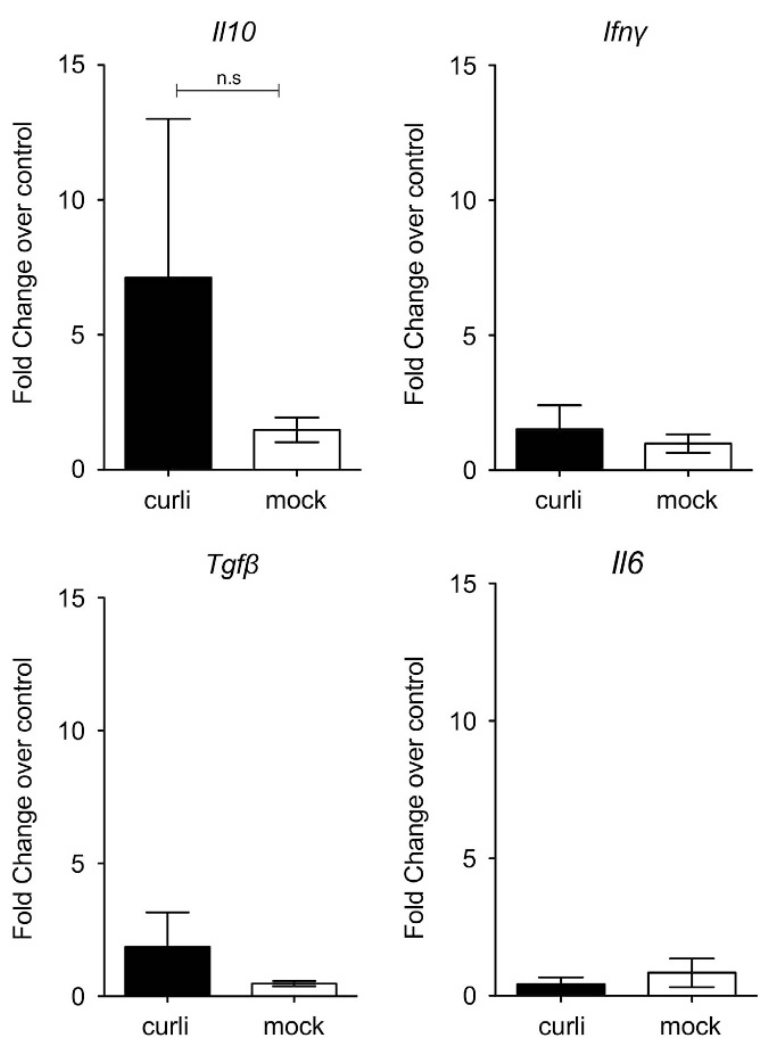

Figure 2. Oral inoculation of curli fibres into mice pretreated with antibiotics did not elicit cytokine expression in the intestine. Female C57BL/6 mice $(n=5)$ were given $0.1 \mathrm{mg}$ of curli fibres orally following a 7-day treatment of mice with a broad-spectrum antibiotic cocktail. Colonic samples were harvested and subjected to RNA extraction after $72 \mathrm{~h}$. Real-time PCR for I/10, Ifn- $\gamma$, Tgf- $\beta$ and $/ / 6$ was performed on mRNA (messenger RNA). important role in eliciting IL-10 production in response to curli fibres and (ii) the immune response against curli fibres in the gut is different from that encountered at systemic sites such as the spleen possibly owing to the presence of specialized immune cell populations previously reported in the gut. ${ }^{55-57}$

One time administration of curli fibres ameliorates the severity of TNBS colitis

Next, we investigated whether oral administration of curli altered mucosal responses in the setting of severe colitis, a condition during which epithelial erosion damages barrier function. We chose TNBS-induced colitis model, an experimental colitis induced in susceptible strains of mice. Although Balb/c, SJL mice are susceptible to TNBS-induced colitis, C57/BL6 mice are resistant and do not develop colitis. ${ }^{42}$ Groups of six to seven Balb/c mice were administered intrarectal instillations of $60 \mu \mathrm{l}$ of $1 \%$ TNBS in $50 \%$ ethanol $(\mathrm{EtOH})$ to induce colitis or received vehicle control (intrarectal instillations of $60 \mu \mathrm{l}$ of $50 \% \mathrm{EtOH}$ ). One day after TNBS administration, mice were mock-treated or treated intragastrically with 0.1 or $0.4 \mathrm{mg}$ purified curli fibres. We compared these groups of mice with another group of mice with TNBS colitis that received an intraperitoneal injection of $0.1 \mathrm{mg}$ of anti-TNFa antibodies as a control, a treatment known to reduce the severity of TNBS colitis. $^{58}$ Mice were euthanized on day 6 after TNBS treatments (Supplementary Figure S5). Weight changes were monitored throughout the experiment (Supplementary Figure S6). Although the mice that received only TNBS enema had a survival rate of $65 \%$, mice that received $0.1 \mathrm{mg}$ curli fibres intragastrically had an $80 \%$ survival rate. Hundred percent mice that received $0.4 \mathrm{mg}$ of curli fibres survived the treatment (Figure 4a). When colonic tissues were scored histopathologically for inflammatory changes, no differences were observed between groups at 2 days after treatments (data not shown). At day 6 post treatment, pathology scores for mice that received $0.1 \mathrm{mg}$ curli fibres intragastrically 1 day post TNBS enema did not differ significantly from the mice that only received TNBS enema. However, single-dose intragastric a
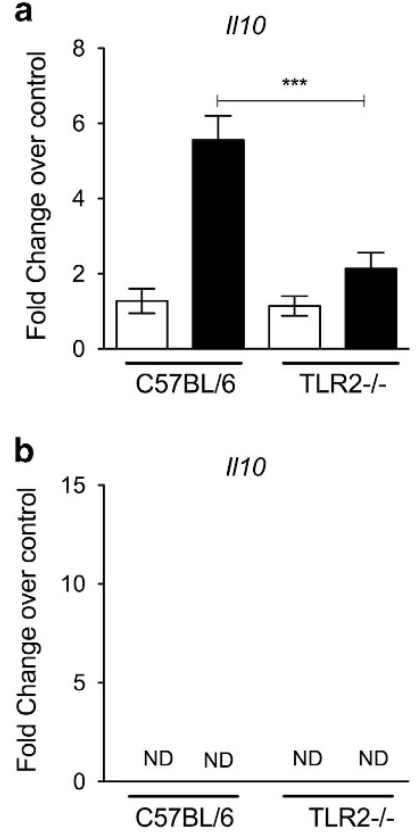
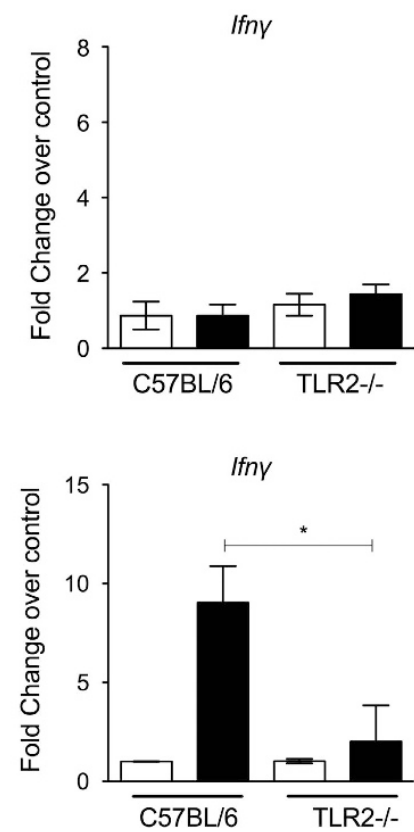

$\operatorname{Tgf} \beta$
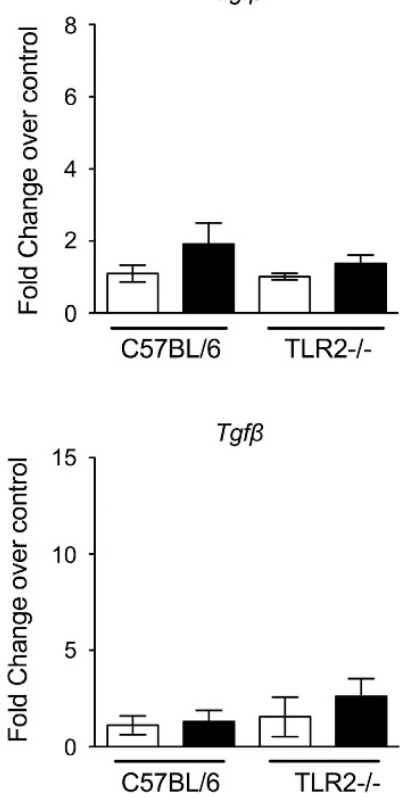

116

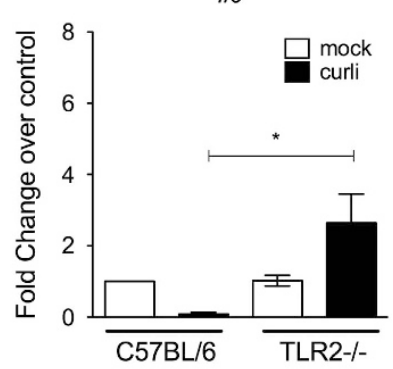

116

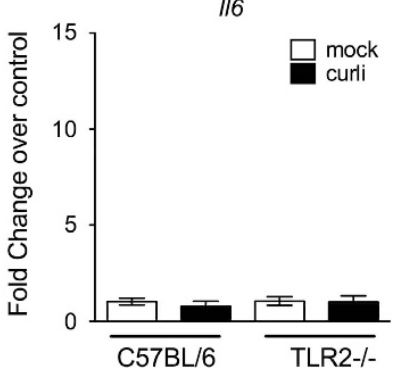

Figure 3. Intraperitoneal injection of curli elicits $/ 110$ expression in intestinal tissue of mice in a TLR2-dependent manner. Age-matched female C57BL/6 and TLR2 - / - mice $(n=5)$ were injected intraperitoneally with $0.1 \mathrm{mg}$ of curli fibres. After $72 \mathrm{~h}$, real-time PCR was performed on intestine (a) and spleen (b) for II10, Ifn- $\gamma$, Tgf- $\beta$ and II6. $\left({ }^{*} P<0.05 ;{ }^{* * *} P<0.001\right)$. 
a

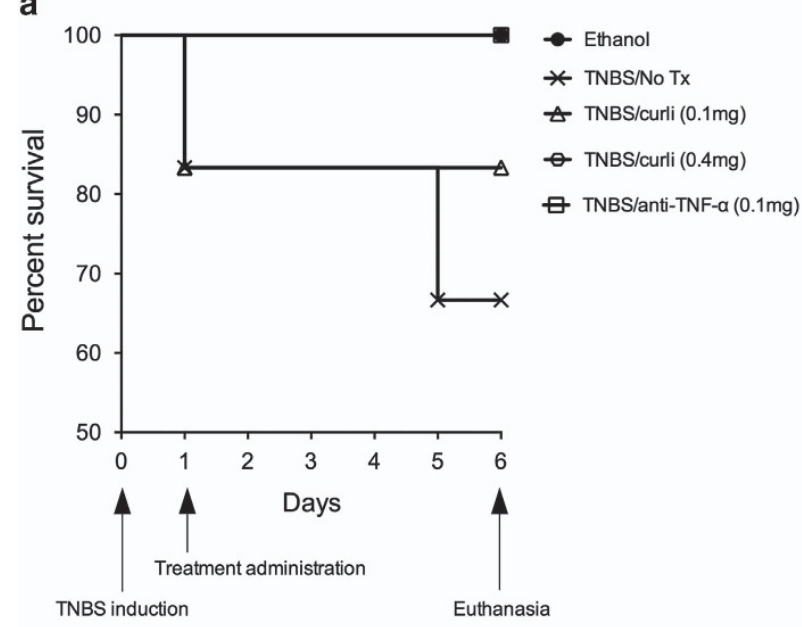

C

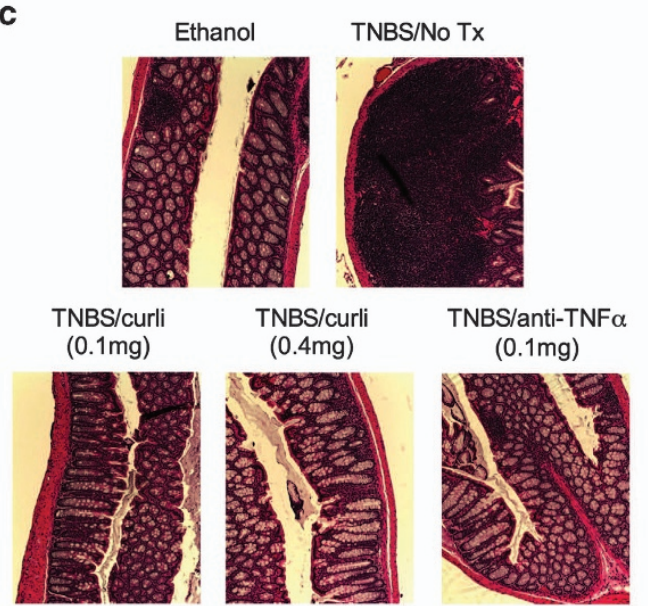

b

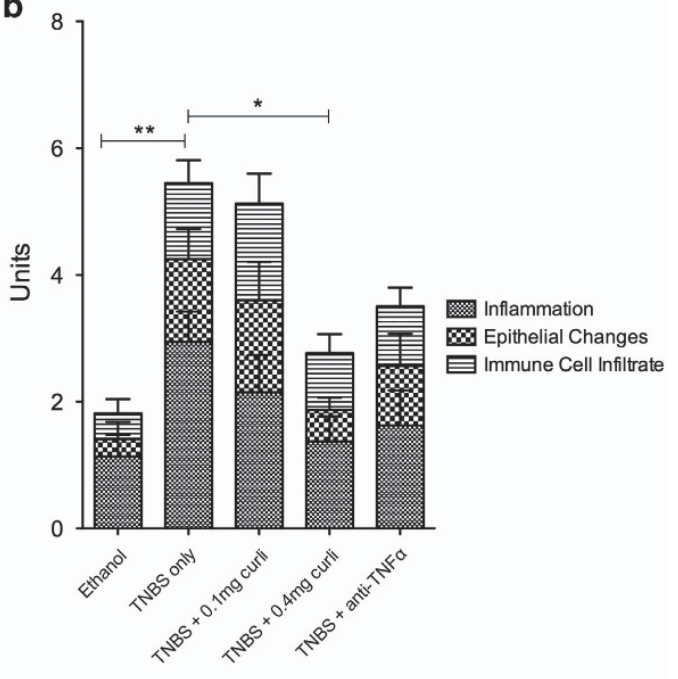

d

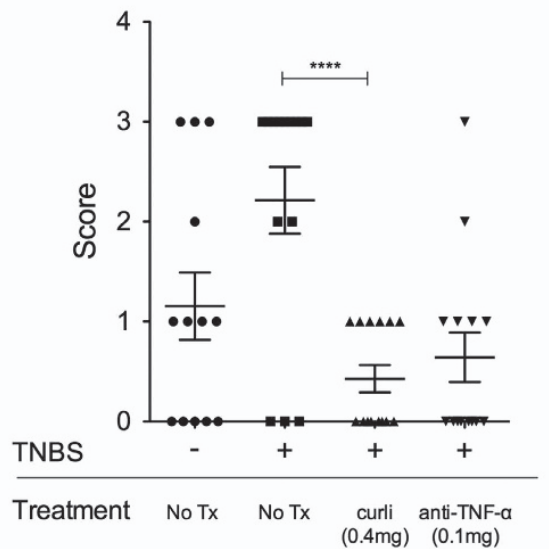

Figure 4. Single treatment with curli fibres ameliorates TNBS colitis. Colitis was induced in 6-8-week female Balb/c $(n=6-7)$ mice by intrarectal instillation of $1 \%$ TNBS in 50\% ethanol or $50 \%$ ethanol as a vehicle control. Day 1 post TNBS enema, mice were administered treatments as follows: $0.1 \mathrm{mg}$ curli (oral), $0.4 \mathrm{mg}$ curli (oral), $0.1 \mathrm{mg}$ anti-TNF $\alpha$ (i.p.) or no treatment. (a) survival $(n=6)$, (b) histopathological scores at day 6 post TNBS induction were plotted and (c) H\&E images were taken. (d) Stool consistency scores were determined at day 3 post TNBS induction. It should be noted that larger areas of immune cell infiltration and lymphoid follicles in the submucosa was determined in the colonic tissue of mice treated with TNBS alone as compared with the groups that received curli treatment $\left({ }^{*} P<0.05 ;{ }^{* *} P<0.01 ;{ }^{* * * * P} P<0.0001\right)$. H\&E, hematoxylin and eosin; i.p., intraperitoneal; TNBS, 2,4,6-trinitrobenzene sulphonic acid; TNF, tumour necrosis factor; Tx, treatment.

treatment with $0.4 \mathrm{mg}$ curli fibres 1 day post TNBS enema decreased the pathology at day 6 and the scores were comparable to the control mice that only received $\mathrm{EtOH}$ vehicle or the mice that received anti-TNFa antibodies post TNBS enema (Figure 4b). Areas of immune cell infiltration and lymphoid follicles in the submucosa were also larger in the colonic tissue of mice treated with TNBS enema alone as compared with the group that received $0.4 \mathrm{mg}$ of curli (Figure 4c).

Faecal matter from each mice in each group was collected at day 2 or day 6 post TNBS enema and scored according to a previously described stool scoring system that looked for stool consistency. ${ }^{59}$ Mice that received only TNBS enema had higher stool scores in their faecal matter than mice that received curli $(0.1$ or $0.4 \mathrm{mg}$ ) or anti-TNFa antibody treatments day 2 post TNBS enema (Figure 4d). No differences were observed at day 6 between any of the groups (Supplementary Figure S7).

Following euthanasia of mice at day 3 or day 6 post TNBS induction, we assayed for the expression of various cytokines in distal colonic tissue via RT-PCR. At day 3 after TNBS treatment, we saw no significant differences in 1110 , Ifng, Tgfb, Tnfa or 116 expression between the different treatment groups (Figure 5a). At day 6 after TNBS treatment, expression of $/ 16$ and Tnfa messenger RNA were increased after TNBS treatment. Interestingly, only administration of curli hightened transcript levels of $I / 10$ by day 6 post TNBS treatment (Figure 5b). An elevated level of Ifng detected in the intestinal mucosa of mice receiving curli (Figure $5 b$ ) did not correlate with the severity of inflammation detected by histopathology. Collectively, these results suggest to us that IL-10 may be one of the critical players in dampening of the inflammatory responses in the mice that received curli fibre treatment following TNBS enema.

\section{DISCUSSION}

Systemic administration of the TLR5 ligand flagellin enhances mucosal barrier function by inducing gene expression in the intestinal epithelium. ${ }^{54}$ Similarly, here we show that systemic administration of curli, a bacterial amyloid that stimulates the TLR2/1 receptor complex, ${ }^{15,29,30}$ induced //10 transcription in the intestinal mucosa. Furthermore, ex vivo exposure of intestinal cells 
a

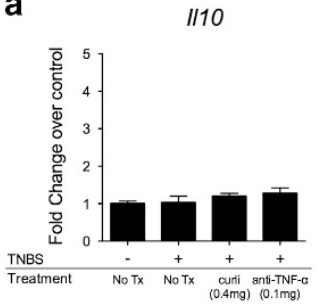

Ifny

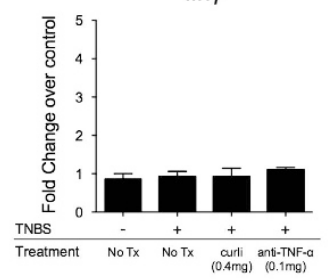

$\operatorname{Tgf\beta }$

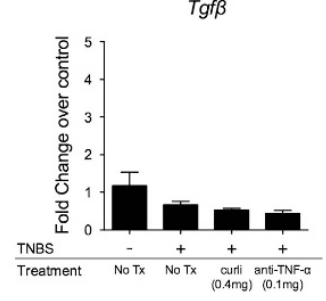

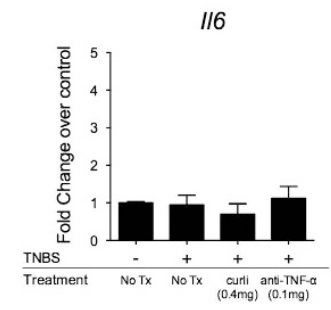

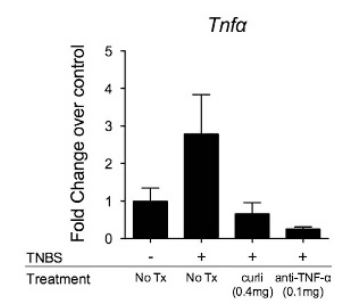

b

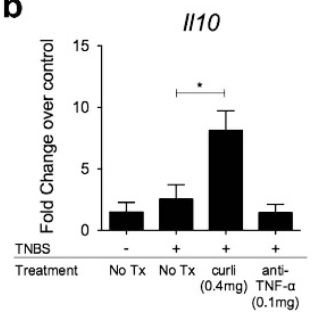

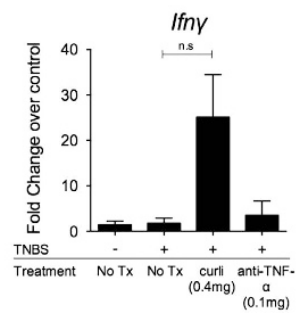
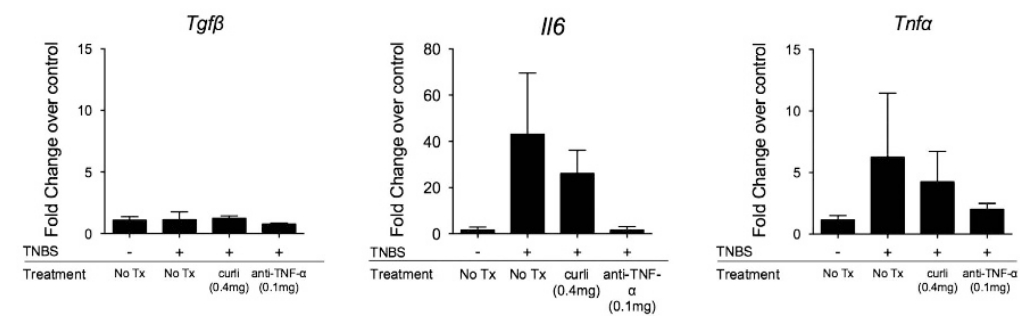

Figure 5. Treatment with curli fibres induce $/ / 10$ expression in the colons of colitic mice. Colonic tissues were harvested from colitic mice at day 3 (a) and 6 (b) post TNBS enema $(n=6)$. Real-time PCR analysis was performed for the expression of II10, Ifn- $\gamma$, Tgf- $\beta$, II6 and Tnfa. Elevated II10 expression was observed at day 6 in the colon ( $\left.{ }^{*} P<0.05\right) ; P>0.05$ (NS). NS, not significant; TNBS, 2,4,6-trinitrobenzene sulphonic acid; Tx, treatment.

to curli resulted in TLR2-dependent IL-10 production. As amyloids are generally resistant to proteolysis, ${ }^{18}$ we investigated whether expression of this anti-inflammatory cytokine could also be induced after oral administration of curli. Remarkably, oral administration of curli elevated $/ 110$ transcript levels in the intestinal mucosa of mice with hapten-induced colitis, whereas no significant increase was observed in conventional mice. These data suggested that induction of $/ 110$ expression by curli is most pronounced when this ligand can enter tissue, either after intraperitoneal injection or when epithelial integrity is impaired during colitis. Similar observations have been made with polysaccharide A (PSA), a TLR2 ligand expressed by commensal Bacteroides fragilis that induces a TLR2-dependent generation of IL-10-producing FoxP3+ T-regulatory cells. ${ }^{60,61}$ Although csg gene cluster, that encodes curli fibres, is found in members of Enterobacteriaceae, the homologues of csg genes were recently determined in the members of Bacteroidetes, Proteobacteria, Firmicutes and Thermodesulfobacteria. ${ }^{25}$ As Bacteroidetes, Proteobacteria and Firmicutes are the most abundant bacteria found in gut microbiota, ${ }^{62}$ these recent studies suggest that numerous bacterial species may express amyloids in the gut. Consistent with this idea, amyloids were recently detected in the biofilms of Bacteriodetes and Firmicutes isolated from sludge wastewater treatment plants. ${ }^{63}$ Nonetheless, further detailed analyses are needed to reveal the abundance of amyloids in the gut communities. In addition, determining the cell populations responsible for IL-10 production in response to curli fibres, e.g., FoxP3+ T-regulatory cells or regulatory monocytes, would reveal novel information in host-microbiota interactions.

Given the importance of IL-10 in acting as an anti-inflammatory molecule dampening inflammation and controlling infections, ${ }^{64,65}$ we explored the potential of curli fibres to reduce intestinal inflammation. Here, we show that single intragastric treatment with curli fibres ameliorates TNBS-induced colitis pathology to a similar degree as treatment with anti-TNFa antibody. ECN has been shown to reduce the severity of colitis and elicit a TLR2dependent production of IL-10 in the gut. ${ }^{49,50}$ However, it is not clear whether the growth conditions used in these studies promoted the expression of curli fibres in EcN.

Epithelial cells and LP macrophages are known to be unresponsive to LPS to avoid excessive inflammation that would be triggered by the members of the microbiota, ${ }^{66,67}$ thus other ligands in E. coli become important in the gut mucosa. Even though purified lipoproteins from $E$. coli also trigger TLR2 activation, when curli fibres are present, it was reported that they are the predominant TLR2/TLR1 ligand on E. coli, probably because the lipoproteins remain buried in the outer membrane, whereas bacterial amyloids are secreted to the cell surface. ${ }^{15}$ Our results showing that the curli fibres from EcN trigger IL-10 production via TLR2 activation suggest that the EcN fibres may be expressed in the $\mathrm{Gl}$ tract regardless of the in vitro growth conditions. However, further studies are needed to test whether curli fibres may be one factor that contributes to the immunomodulatory effects of EcN in the gut.

Recently, it was determined that curli fibres complex with extracellular DNA in biofilms. Even though curli is subjected to DNAse and RNase treatment during purification, extracellular DNA embedded into the amyloid structure remains intact. Thus, it is possible that the extracellular DNA associated with the purified curli preparations may also be stimulating the immune system via the cytosolic DNA sensors leading to immune homeostasis in the gut. ${ }^{68}$ TLR9 recognises bacterial double-stranded DNA. ${ }^{51}$ Polymorphisms in TLR2, NOD2, NLRP3 and TLR9 have all been identified as risk factors for developing inflammatory bowel disease. ${ }^{69-71}$ Thus, the success of curli treatment in colitis may be owing to the activation of multiple receptors with this bacterial component including TLR2 and NLRP3 and possibly TLR9. However, detailed analysis will reveal the pathways and receptors activated by oral curli administration in the gut.

Oral administration of curli fibres represents an exciting new strategy to ameliorate intestinal inflammation. Nevertheless, the translocation of curli fibres from the gut into the systemic circulation must carefully be monitored as amyloids are associated with many complex human diseases and considered pathogenic. $^{72}$ It has previously been determined that systemic presence of curli fibres may lead to the production of proinflammatory cytokines, nitric oxide and autoantibodies. ${ }^{68,73,74}$ However, it is not known whether significant amounts of lumenal curli would cross into the LP and the systemic sites during colitis as phagocytes are capable of digesting and clearing amyloids. Overall, the interaction of gut mucosa with bacterial biofilm material is an intriguing line of research that needs to be explored further. 


\section{ACKNOWLEDGEMENTS}

The authors thank Drs Andreas Baumler and Alex Tsygankov for their critical reading of this article. This study was supported by the National Institutes of Health, National Institute of Allergy and Infectious Diseases Grant 1R03Al107434, 1R21Al105370 and by Biostrategy Partners Pharma Germinator Award.

\section{CONTRIBUTIONS}

GOO performed the experiments related to the manuscript. GJR assisted GOO in the colitis experiments. AJPK-S scored the tissue slides for histopathology. MG provided the E. coli Nissle 1917 strain and edited the manuscript. SGB confirmed the expression of curli fibres by E. coli Nissle 1917. CM and CH helped with the TNBSinduced colitis experiments and the interpretation of the data. RPW helped design the experiments and edited the figures of the manuscript. $C T$ designed and oversaw all the experimental work. CT and GOO wrote the manuscript, which was further edited by all the authors. All the authors approved the final manuscript.

\section{COMPETING INTERESTS}

The authors declare no conflict of interest.

\section{REFERENCES}

1 Turnbaugh PJ, Ley RE, Hamady M, Fraser-Liggett CM, Knight R, Gordon Jl. The human microbiome project. Nature 2007; 449: 804-810.

2 Frank DN, St Amand AL, Feldman RA, Boedeker EC, Harpaz N, Pace NR. Molecularphylogenetic characterization of microbial community imbalances in human inflammatory bowel diseases. Proc Natl Acad Sci USA 2007; 104: 13780-13785.

3 Gill SR, Pop M, Deboy RT, Eckburg PB, Turnbaugh PJ, Samuel BS et al. Metagenomic analysis of the human distal gut microbiome. Science 2006; 312: 1355-1359.

4 Abraham C, Medzhitov R. Interactions between the host innate immune system and microbes in inflammatory bowel disease. Gastroenterology 2011; 140: 1729-1737.

5 Cario E. Barrier-protective function of intestinal epithelial Toll-like receptor 2. Mucosal Immunol 2008; 1(Suppl 1): S62-S66.

6 Cario E, Gerken G, Podolsky DK. Toll-like receptor 2 controls mucosal inflammation by regulating epithelial barrier function. Gastroenterology 2007; 132: 1359-1374.

7 Cario E, Gerken G, Podolsky DK. Toll-like receptor 2 enhances ZO-1-associated intestinal epithelial barrier integrity via protein kinase C. Gastroenterology 2004; 127: 224-238.

8 Rakoff-Nahoum S, Paglino J, Eslami-Varzaneh F, Edberg S, Medzhitov R. Recognition of commensal microflora by toll-like receptors is required for intestinal homeostasis. Cell 2004; 118: 229-241.

9 Gibson DL, Ma C, Rosenberger CM, Bergstrom KS, Valdez Y, Huang JT et al. Tolllike receptor 2 plays a critical role in maintaining mucosal integrity during Citrobacter rodentium-induced colitis. Cell Microbiol 2008; 10: 388-403.

10 Horng T, Barton GM, Flavell RA, Medzhitov R. The adaptor molecule TIRAP provides signalling specificity for Toll-like receptors. Nature 2002; 420: 329-333.

11 Yamamoto M, Sato S, Hemmi H, Sanjo H, Uematsu S, Kaisho T et al. Essential role for TIRAP in activation of the signalling cascade shared by TLR2 and TLR4. Nature 2002; 420: 324-329.

12 Li J, Lee DS, Madrenas J. Evolving bacterial envelopes and plasticity of TLR2dependent responses: basic research and translational opportunities. Front Immunol 2013; 4: 347.

13 Kuhn R, Lohler J, Rennick D, Rajewsky K, Muller W. Interleukin-10-deficient mice develop chronic enterocolitis. Cell 1993; 75: 263-274.

14 Akira S, Takeda K. Toll-like receptor signalling. Nat Rev Immunol 2004; 4: 499-511.

15 Tukel C, Nishimori JH, Wilson RP, Winter MG, Keestra AM, van Putten JP et al. Tolllike receptors 1 and 2 cooperatively mediate immune responses to curli, a common amyloid from enterobacterial biofilms. Cell Microbiol 2010; 12: 1495-1505.

16 Blanco LP, Evans ML, Smith DR, Badtke MP, Chapman MR. Diversity, biogenesis and function of microbial amyloids. Trends Microbiol 2011; 20: 66-73.

17 Chapman MR, Robinson LS, Pinkner JS, Roth R, Heuser J, Hammar M et al. Role of Escherichia coli curli operons in directing amyloid fiber formation. Science 2002; 295: 851-855.

18 Hufnagel DA, Tukel C, Chapman MR. Disease to dirt: the biology of microbial amyloids. PLoS Pathog 2013; 9: e1003740.

19 Hung C, Zhou Y, Pinkner JS, Dodson KW, Crowley JR, Heuser J et al. Escherichia coli biofilms have an organized and complex extracellular matrix structure. MBio 2014; 4: e00645-13.
20 Romling U, Bian Z, Hammar M, Sierralta WD, Normark S. Curli fibers are highly conserved between Salmonella typhimurium and Escherichia coli with respect to operon structure and regulation. J Bacteriol 1998; 180: 722-731.

21 Prigent-Combaret $C$, Brombacher E, Vidal O, Ambert A, Lejeune P, Landini P et al. Complex regulatory network controls initial adhesion and biofilm formation in Escherichia coli via regulation of the csgD gene. J Bacteriol 2001; 183: 7213-7223.

22 Hammer ND, Schmidt JC, Chapman MR. The curli nucleator protein, CsgB, contains an amyloidogenic domain that directs CsgA polymerization. Proc Natl Acad Sci USA 2007; 104: 12494-12499.

23 Bian Z, Normark S. Nucleator function of CsgB for the assembly of adhesive surface organelles in Escherichia coli. EMBO J 1997; 16: 5827-5836.

24 Hammar M, Bian Z, Normark S. Nucleator-dependent intercellular assembly of adhesive curli organelles in Escherichia coli. Proc Natl Acad Sci USA 1996; 93: 6562-6566.

25 Dueholm MS, Albertsen M, Otzen D, Nielsen PH. Curli functional amyloid systems are phylogenetically widespread and display large diversity in operon and protein structure. PLoS ONE 2012; 7: e51274.

26 Gualdi L, Tagliabue L, Bertagnoli S, lerano T, De Castro C, Landini P. Cellulose modulates biofilm formation by counteracting curli-mediated colonization of solid surfaces in Escherichia coli. Microbiology 2008; 154(Pt 7): 2017-2024.

27 Gophna U, Barlev M, Seijffers R, Oelschlager TA, Hacker J, Ron EZ. Curli fibers mediate internalization of Escherichia coli by eukaryotic cells. Infect Immun 2001; 69: 2659-2665.

28 Barak JD, Gorski L, Naraghi-Arani P, Charkowski AO. Salmonella enterica virulence genes are required for bacterial attachment to plant tissue. Appl Environ Microbiol 2005; 71: 5685-5691.

29 Tukel C, Raffatellu M, Humphries AD, Wilson RP, Andrews-Polymenis HL, Gull T et al. CsgA is a pathogen-associated molecular pattern of Salmonella enterica serotype Typhimurium that is recognized by Toll-like receptor 2. Mol Microbiol 2005; 58: 289-304.

30 Tukel C, Wilson RP, Nishimori JH, Pezeshki M, Chromy BA, Baumler AJ. Responses to amyloids of microbial and host origin are mediated through Toll-like receptor 2. Cell Host Microbe 2009; 6: 45-53.

31 Oppong GO, Rapsinski GJ, Newman TN, Nishimori JH, Biesecker SG, Tukel C. Epithelial cells augment barrier function via activation of the Toll-like receptor 2/ phosphatidylinositol 3-kinase pathway upon recognition of Salmonella enterica Serovar Typhimurium curli fibrils in the gut. Infect Immun 2013; 81: 478-486.

32 Rapsinski GJ, Wynosky-Dolfi MA, Oppong GO, Tursi SA, Wilson RP, Brodsky IE et al. Toll-like receptor 2 and NLRP3 cooperate to recognize a functional bacterial amyloid, curli. Infect Immun 2014; 83: 693-701.

33 Kretschmer D, Gleske AK, Rautenberg M, Wang R, Koberle M, Bohn E et al. Human formyl peptide receptor 2 senses highly pathogenic Staphylococcus aureus. Cell Host Microbe 2010; 7: 463-473.

34 Schwartz K, Syed AK, Stephenson RE, Rickard AH, Boles BR. Functional amyloids composed of phenol soluble modulins stabilize Staphylococcus aureus biofilms. PLoS Pathog 2012; 8: e1002744.

35 Liang TS, Wang JM, Murphy PM, Gao JL. Serum amyloid A is a chemotactic agonist at FPR2, a low-affinity $\mathrm{N}$-formylpeptide receptor on mouse neutrophils. Biochem Biophys Res Commun 2000; 270: 331-335

36 Cui $Y H$, Le Y, Zhang X, Gong W, Abe K, Sun R et al. Up-regulation of FPR2, a chemotactic receptor for amyloid beta 1-42 (A beta 42), in murine microglial cells by TNF alpha. Neurobiol Dis 2002; 10: 366-377.

37 Barnhart MM, Chapman MR. Curli biogenesis and function. Annu Rev Microbiol 2006; 60: 131-147.

38 Nissle A. [Sanitation of intestinal flora as a prophylactic measure]. Medizinische 1957; 10: 82-85.

39 Raffatellu M, Chessa D, Wilson RP, Dusold R, Rubino S, Baumler AJ. The Vi capsular antigen of Salmonella enterica serotype Typhi reduces Toll-like receptor-dependent interleukin-8 expression in the intestinal mucosa. Infect Immun 2005; 73: 3367-3374.

40 Lim JY, May JM, Cegelski L. Dimethyl sulfoxide and ethanol elicit increased amyloid biogenesis and amyloid-integrated biofilm formation in Escherichia coli. Appl Environ Microbiol 2012; 78: 3369-3378.

41 Collinson SK, Emody L, Muller KH, Trust TJ, Kay WW. Purification and characterization of thin, aggregative fimbriae from Salmonella enteritidis. J Bacteriol 1991; 173: $4773-4781$.

42 Scheiffele F, Fuss IJ. Induction of TNBS colitis in mice. Curr Protoc Immunol 2002; Chapter 15: Unit 15.19.

43 Kong W, Yen JH, Ganea D. Docosahexaenoic acid prevents dendritic cell maturation, inhibits antigen-specific Th1/Th17 differentiation and suppresses experimental autoimmune encephalomyelitis. Brain Behav Immun 2011; 25: 872-882.

44 Overbergh L, Giulietti A, Valckx D, Decallonne R, Bouillon R, Mathieu C. The use of real-time reverse transcriptase $P C R$ for the quantification of cytokine gene expression. J Biomol Tech 2003; 14: 33-43. 
45 Wilson RP, Raffatellu M, Chessa D, Winter SE, Tukel C, Baumler AJ. The Vi-capsule prevents Toll-like receptor 4 recognition of Salmonella. Cell Microbiol 2008; 10: 876-890.

46 Roux CM, Rolan HG, Santos RL, Beremand PD, Thomas TL, Adams LG et al. Brucella requires a functional Type IV secretion system to elicit innate immune responses in mice. Cell Microbiol 2007; 9: 1851-1869.

47 Nishimori JH, Newman TN, Oppong GO, Rapsinski GJ, Yen JH, Biesecker SG et al. Microbial amyloids induce IL-17A/IL-22 responses via Toll-like Receptor 2 activation in the intestinal mucosa. Infect Immun 2012; 80: 4398-4408.

48 Rapsinski GJ, Newman TN, Oppong GO, van Putten JP, Tukel C. CD14 protein acts as an adaptor molecule for the immune recognition of Salmonella curli fibers. J Biol Chem 2013; 288: 14178-14188.

49 Sturm A, Rilling K, Baumgart DC, Gargas K, Abou-Ghazale T, Raupach B et al. Escherichia coli Nissle 1917 distinctively modulates T-cell cycling and expansion via toll-like receptor 2 signaling. Infect Immun 2005; 73: 1452-1465.

50 Grabig A, Paclik D, Guzy C, Dankof A, Baumgart DC, Erckenbrecht J et al. Escherichia coli strain Nissle 1917 ameliorates experimental colitis via toll-like receptor 2- and toll-like receptor 4-dependent pathways. Infect Immun 2006; 74 4075-4082.

51 Hemmi H, Takeuchi O, Kawai T, Kaisho T, Sato S, Sanjo H et al. A Toll-like receptor recognizes bacterial DNA. Nature 2000; 408: 740-745.

52 Zhou Y, Smith DR, Hufnagel DA, Chapman MR. Experimental manipulation of the microbial functional amyloid called curli. Methods Mol Biol 2013; 966: 53-75.

53 Ayres JS, Trinidad NJ, Vance RE. Lethal inflammasome activation by a multidrug resistant pathobiont upon antibiotic disruption of the microbiota. Nat Med 2012 18: 799-806.

54 Kinnebrew MA, Ubeda C, Zenewicz LA, Smith N, Flavell RA, Pamer EG. Bacterial flagellin stimulates Toll-like receptor 5-dependent defense against vancomycinresistant Enterococcus infection. J Infect Dis 2010; 201: 534-543.

55 Round JL, Mazmanian SK. The gut microbiota shapes intestinal immune responses during health and disease. Nat Rev Immunol 2009; 9: 313-323.

56 Coombes JL, Maloy KJ. Control of intestinal homeostasis by regulatory T cells and dendritic cells. Semin Immunol 2007; 19: 116-126.

57 Coombes JL, Siddiqui KR, Arancibia-Carcamo CV, Hall J, Sun CM, Belkaid Y et al. A functionally specialized population of mucosal CD103+ DCs induces Foxp3+ regulatory $\mathrm{T}$ cells via a TGF-beta and retinoic acid-dependent mechanism. J Exp Med 2007; 204: 1757-1764.

58 Neurath MF, Fuss I, Pasparakis M, Alexopoulou L, Haralambous S, Meyer zum Buschenfelde $\mathrm{KH}$ et al. Predominant pathogenic role of tumor necrosis factor in experimental colitis in mice. Eur J Immunol 1997; 27: 1743-1750.

59 Wirtz S, Neufert C, Weigmann B, Neurath MF. Chemically induced mouse models of intestinal inflammation. Nat Protoc 2007; 2: 541-546.

60 Cohen-Poradosu R, McLoughlin RM, Lee JC, Kasper DL. Bacteroides fragilisstimulated interleukin-10 contains expanding disease. J Infect Dis 2011; 204 363-371.
61 Round JL, Mazmanian SK. Inducible Foxp3+ regulatory T-cell development by a commensal bacterium of the intestinal microbiota. Proc Natl Acad Sci USA 2010; 107: 12204-12209.

62 Eckburg PB, Bik EM, Bernstein CN, Purdom E, Dethlefsen L, Sargent M et al. Diversity of the human intestinal microbial flora. Science 2005; 308: 1635-1638.

63 Larsen P, Nielsen JL, Otzen D, Nielsen PH. Amyloid-like adhesins produced by flocforming and filamentous bacteria in activated sludge. Appl Environ Microbiol 2008; 74: $1517-1526$.

64 Donnelly RP, Dickensheets H, Finbloom DS. The interleukin-10 signal transduction pathway and regulation of gene expression in mononuclear phagocytes. Interferon Cytokine Res 1999; 19: 563-573.

65 Moore KW, de Waal Malefyt R, Coffman RL, O'Garra A. Interleukin-10 and the interleukin-10 receptor. Annu Rev Immunol 2001; 19: 683-765.

66 Melmed G, Thomas LS, Lee N, Tesfay SY, Lukasek K, Michelsen KS et al. Human intestinal epithelial cells are broadly unresponsive to Toll-like receptor 2-dependent bacterial ligands: implications for host-microbial interactions in the gut. J Immunol 2003; 170: 1406-1415.

67 Smith PD, Smythies LE, Mosteller-Barnum M, Sibley DA, Russell MW, Merger M et al. Intestinal macrophages lack CD14 and CD89 and consequently are downregulated for LPS- and IgA-mediated activities. J Immunol 2001; 167: 2651-2656.

68 Gallo PM, Rapsinski GJ, Wilson RP, Oppong GO, Sriram U, Goulian M et al. Amyloid-DNA composites of bacterial biofilms stimulate autoimmunity. Immunity 2015; 42: 1171-1184.

69 Mowat AM, Bain CC. Mucosal macrophages in intestinal homeostasis and inflammation. J. Innate Immun 2011; 3: 550-564.

70 Pierik M, Joossens S, Van Steen K, Van Schuerbeek N, Vlietinck R, Rutgeerts P et al. Toll-like receptor- $1,-2$, and -6 polymorphisms influence disease extension in inflammatory bowel diseases. Inflamm Bowel Dis 2006; 12: 1-8.

71 Zaki MH, Lamkanfi M, Kanneganti TD. The Nlrp3 inflammasome: contributions to intestinal homeostasis. Trends Immunol 2011; 32: 171-179.

72 Rambaran RN, Serpell LC. Amyloid fibrils: abnormal protein assembly. Prion 2008 2: 112-117.

73 Bian Z, Yan ZQ, Hansson GK, Thoren P, Normark S. Activation of inducible nitric oxide synthase/nitric oxide by curli fibers leads to a fall in blood pressure during systemic Escherichia coli infection in mice. J Infect Dis 2001; 183: 612-619.

74 Bian Z, Brauner A, Li Y, Normark S. Expression of and cytokine activation by Escherichia coli curli fibers in human sepsis. J Infect Dis 2000; 181: 602-612.

\begin{abstract}
(c) (i)
This work is licensed under a Creative Commons Attribution 4.0 International License. The images or other third party material in this article are included in the article's Creative Commons license, unless indicated otherwise in the credit line; if the material is not included under the Creative Commons license, users will need to obtain permission from the license holder to reproduce the material. To view a copy of this license, visit http://creativecommons.org/licenses/ by/4.0/
\end{abstract}

Supplemental Information accompanies the paper on the npj Biofilms and Microbiomes website (http://www.nature.com/npjbiofilms) 